\title{
Intramolecular Endo-Dig Hydrosilylation Catalyzed by Ruthenium: Evidence for a New Mechanistic Pathway
}

\author{
Barry M. Trost and Zachary T. Ball \\ Department of Chemistry, Stanford University, Stanford, CA 94305-5080.
}

\section{Supplemental Information}

All reactions were performed under argon atmosphere unless otherwise indicated. Methylene chloride $\left(\mathrm{CH}_{2} \mathrm{Cl}_{2}\right)$ was purified on an alumina column solvent purification system. Dimethylformamide (DMF) was purchased from Aldrich and used as received. Pyridine was distilled from calcium hydride. All other reagents were purchased and used as received. $\left[\mathrm{Cp}^{*} \mathrm{Ru}(\mathrm{MeCN})_{3}\right] \mathrm{PF}_{6}$ was synthesized as described by Schenk. ${ }^{1}$

Flash chromatography was performed with EM Science silica gel (0.040-0.063 _m grade) or 200 mesh Florisil (Aldrich). Analytical thin layer chromatography was performed with 0.2mm coated commercial silica gel plates (E. Merck, DC Plastifolien, kieselgel $60 \mathrm{~F}_{254}$ ).

Proton and broad-band decoupled ${ }^{13} \mathrm{C}$ nuclear magnetic resonance data were acquired on a Varian GEM-300, Mercury-400 or Unity Inova-500 spectrometer as indicated. Chemical shifts are reported in ppm relative to TMS or $\mathrm{C}_{6} \mathrm{D}_{6}$ as an internal standard.

Infrared (IR) data were recorded in sodium chloride plates on PerkinElmer Paragon 500 FT-IR spectrometer. Gas chromatographic and GC-MS analyses were performed on a Hewlett-Packard 6890 Series gas chromatograph with attached HP 5973 Series mass detection.

General Procedure for Intramolecular Hydrosilylation

( \pm )-(1R,2S)-(2,2-Dimethyl-3-(3-phenyl-propyl)-4a,5, 6, 7,8,8a-hexahydro-2H-

benzo[e][1,2]oxasiline 


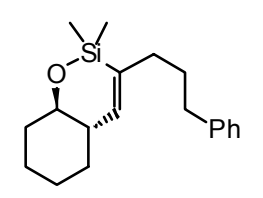

A round-bottomed flask [Note: it is important to use a rather large flask to allow removal of the excess silane under vacuum without bumping] was charged with $( \pm)-(1 S, 2 S)-2-(5-p h e n y l-1-$ propynyl)-cyclohexanol $(123 \mu \mathrm{L}, 0.50 \mathrm{mmol})$ under Ar at rt. To the neat alcohol was added 1,1,3,3-tetramethyldisilazane $(270 \mu \mathrm{L}, 1.5 \mathrm{mmol})$ and the flask heated to $50{ }^{\circ} \mathrm{C}$ for $2 \mathrm{hr}$. Next, the flask was cooled to ambient temperature and placed under vacuum (c.a. $1 \mathrm{mmHg}$ ) for $45 \mathrm{~min}$ to remove the excess silazane. An Ar atmosphere was then re-introduced and the residue taken up in $\mathrm{CH}_{2} \mathrm{Cl}_{2}(1.0 \mathrm{~mL})$. The flask was cooled to $0{ }^{\circ} \mathrm{C}$ and solid $\left[\mathrm{Cp}{ }^{*} \mathrm{Ru}(\mathrm{MeCN})_{3}\right] \mathrm{PF}_{6}(2.5 \mathrm{mg}$, $0.005 \mathrm{mmol}$ ) was added to the solution. The flask was allowed to warm to $\mathrm{rt}$, and after $15 \mathrm{~min}$, the solution was diluted with ether $(5 \mathrm{~mL})$ and filtered through a short plug of florisil, washing with additional ether $(15 \mathrm{~mL})$. The volatile components were then removed under reduced pressure and the resulting residue purified on a florisil column (eluent 30:1 pet. ether: ether) to afford $130 \mathrm{mg}(85 \%)$ of the desired silacycle as a colorless oil. NMR analysis of all products (including cross-coupling product) shown indicated complete isomeric purity. A conservative estimate of $\geq 97: 3$ isomeric purity for all products is in line with the uncertainty in NMR measurements.

${ }^{1} \mathrm{H}$ NMR $\left(300 \mathrm{MHz}, \mathrm{CDCl}_{3}\right) \delta 7.28(\mathrm{dd}, \mathrm{J}=7.6,7.6 \mathrm{~Hz}, 2 \mathrm{H}), 7.15-7.19(\mathrm{~m}, 3 \mathrm{H}), 6.03(\mathrm{~s}, 1 \mathrm{H})$, 3.38 (ddd, J = 10.0, 10.0, $3.9 \mathrm{~Hz}, 1 \mathrm{H}), 2.59$ (t, J = 7.7 Hz, 2H), $2.10(\mathrm{t}, \mathrm{J}=7.3 \mathrm{~Hz}, 2 \mathrm{H}), 1.96(\mathrm{~m}$, $2 \mathrm{H}), 1.65-1.80(\mathrm{~m}, 5 \mathrm{H}), 1.00-1.42(\mathrm{~m}, 4 \mathrm{H}), 0.19(\mathrm{~s}, 6 \mathrm{H}) .{ }^{13} \mathrm{C} \mathrm{NMR}\left(75 \mathrm{MHz}, \mathrm{CDCl}_{3}\right) \delta 146.2$, 142.5, 138.5, 128.4, 128.3, 125.7, 76.0, 44.7, 35.7, 35.0, 34.7, 32.0, 31.2, 25.8, 25.0, -0.7. IR (thin film) 3027 (w), 2930 (s), 2855, 1603 (w), 1496 (w), 1449, 1248, 1064, 948, 882, 828, 785 $\mathrm{cm}^{-1}$. Anal. calc. for $\mathrm{C}_{19} \mathrm{H}_{28} \mathrm{OSi}$ : C, 75.94; H, 9.39; Found: C, 75.82; H, 9.30.

6-Ethyl-2,2,3-trimethyl-5,6-dihydro-2H-[1,2]oxasiline

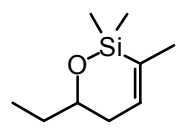


$\mathrm{R}_{\mathrm{f}} 0.59\left(10: 1\right.$ pet. ether: EtOAc). ${ }^{1} \mathrm{H}$ NMR $\left(300 \mathrm{MHz}, \mathrm{CDCl}_{3}\right) \delta 6.30(\mathrm{~m}, 1 \mathrm{H}), 3.74(\mathrm{~m}, 1 \mathrm{H}), 2.10$ (m, 1H), $1.71(\mathrm{~d}, J=1.7 \mathrm{~Hz}, 3 \mathrm{H}), 1.43-1.62(\mathrm{~m}, 2 \mathrm{H}), 0.92$ (d, $J=7.4 \mathrm{~Hz}, 3 \mathrm{H}), 0.18$ (s, 3H), 0.17 (s, 3H). ${ }^{13} \mathrm{C}$ NMR $\left(300 \mathrm{MHz}, \mathrm{CDCl}_{3}\right) \delta 139.5,134.8,73.2,35.5,30.6,20.2,10.0,-1.8,-1.9$. IR (thin film) 2963, 1614, 1440, $1253 \mathrm{~cm}^{-1}$.

3-Heptyl-2,2,7-trimethyl-2,5,6,7-tetrahydro-[1,2]oxasilepine

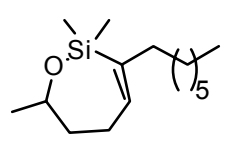

${ }^{1} \mathrm{H}$ NMR $\left(500 \mathrm{MHz}, \mathrm{CDCl}_{3}\right) \delta 6.22(\mathrm{t}, J=6.3 \mathrm{~Hz}, 1 \mathrm{H}), 4.02(\mathrm{~m}, 1 \mathrm{H}), 2.42(\mathrm{~m}, 1 \mathrm{H}), 2.14(\mathrm{~m}$, 1H), 2.05 (m, 1H), 1.99 (m, 1H), 1.74 (ddt, $J=14.2,10.7,3.7 \mathrm{~Hz}, 1 \mathrm{H}), 1.60$ (m, 1H), 1.22-1.32 $(\mathrm{m}, 16 \mathrm{H}), 1.20(\mathrm{~d}, J=6.4 \mathrm{~Hz}, 3 \mathrm{H}), 0.88(\mathrm{t}, J=7.0 \mathrm{~Hz}, 3 \mathrm{H}), 0.24(\mathrm{~s}, 3 \mathrm{H}), 0.18(\mathrm{~s}, 3 \mathrm{H}) .{ }^{13} \mathrm{C} \mathrm{NMR}$ $\left(75 \mathrm{MHz}, \mathrm{CDCl}_{3}\right) \delta$ 142.6, 140.4, 68.7, 36.9, 36.6, 31.9, 30.2, 29.6 (2), 29.5 (2), 29.3, 26.7, 23.9, 22.7, 14.1, 0.54, -1.0. Anal. calc. for $\mathrm{C}_{17} \mathrm{H}_{34} \mathrm{OSi}$ : C, 72.27; H, 12.13; Found: $\mathrm{C}, 72.37$; H, 11.96 .

6,6,7-Trimethyl-3,4,4a,6,9,9a-hexahydro-1H-5-oxa-6-sila-benzocyclohepten-2-one 2-ethylene ketal

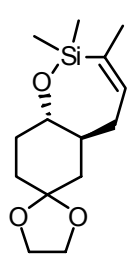

$\mathrm{R}_{\mathrm{f}} 0.48$ (4:1 pet. ether: EtOAc). ${ }^{1} \mathrm{H}$ NMR $\left(300 \mathrm{MHz}, \mathrm{CDCl}_{3}\right) \delta 6.23(\mathrm{~m}, 1 \mathrm{H}), 3.43-3.57(\mathrm{~m}, 5 \mathrm{H})$, $1.88-2.28(\mathrm{~m}, 5 \mathrm{H}), 1.71(\mathrm{~s}, 3 \mathrm{H}), 1.33-1.68(\mathrm{~m}, 4 \mathrm{H}), 1.32(\mathrm{~s}, 3 \mathrm{H}), 0.24(\mathrm{~s}, 3 \mathrm{H}) .{ }^{13} \mathrm{C}$ NMR $(75$ $\left.\mathrm{MHz}, \mathrm{CDCl}_{3}\right) \delta 141.2,138.2,108.6,78.8,64.33,64.28,43.1,42.0,37.0,33.7,33.1,22.3,0.85$, 1.1. IR (thin film) 2946 (s), 1622 (w), 1145, 1250, 1148 (s), 1119 (s), 1078 (s), 932, 825, $779 \mathrm{~cm}^{-}$ ${ }^{1}$. HRMS—EI (m/z): [M] $]^{+}$calcd for $\mathrm{C}_{14} \mathrm{H}_{24} \mathrm{O}_{3} \mathrm{Si}$ : 268.1495; found: 268.1496 .

Ethyl 2,2-Dimethyl-3-pentyl-5,6-dihydro-2H-[1,2]oxasiline-6-carboxylate 


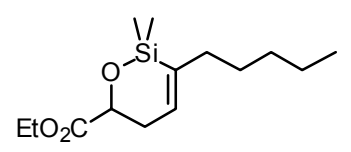

$\mathrm{R}_{\mathrm{f}} 0.29$ (10:1 pet. ether: EtOAc). ${ }^{1} \mathrm{H}$ NMR $\left(400 \mathrm{MHz}, \mathrm{C}_{6} \mathrm{D}_{6}\right) \delta 6.16(\mathrm{~m}, 1 \mathrm{H}), 4.52(\mathrm{dd}, J=7.5$, $4.3 \mathrm{~Hz}, 1 \mathrm{H}), 3.94$ (q, $J=7.2 \mathrm{~Hz}, 2 \mathrm{H}), 2.51(\mathrm{~m}, 1 \mathrm{H}), 2.39(\mathrm{~m}, 1 \mathrm{H}), 2.02(\mathrm{~m}, 2 \mathrm{H}), 1.18-1.37$ (m, $6 \mathrm{H}), 0.91(\mathrm{t}, J=7.2 \mathrm{~Hz}, 3 \mathrm{H}), 0.89$ (t, $J=7.3 \mathrm{~Hz}, 3 \mathrm{H}), 0.39$ (s, 3H), 0.21 (s, 3H). ${ }^{13} \mathrm{C}$ NMR $(100$ $\left.\mathrm{MHz}, \mathrm{C}_{6} \mathrm{D}_{6}\right) \delta 172.3,141.4,137.1,71.3,60.6,35.7,32.9,31.9,29.4,22.8,14.3,14.2,0.1,-0.3$. IR (thin film) 2958, 2927, 1754, 1733 (w), 1251, 1188, 832, $784 \mathrm{~cm}^{-1}$. Anal. calc. for $\mathrm{C}_{14} \mathrm{H}_{26} \mathrm{O}_{3} \mathrm{Si}$ : C, 62.18; H, 9.69; Found: C, 62.28; H, 9.53.

3-Benzyloxymethyl-2,2,5,5,7-pentamethyl-2,5,6,7-tetrahydro-[1,2]oxasilepine

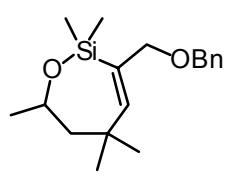

$\mathrm{R}_{\mathrm{f}} 0.41$ (10:1 pet. ether: ether). ${ }^{1} \mathrm{H}$ NMR $\left(400 \mathrm{MHz}, \mathrm{C}_{6} \mathrm{D}_{6}\right) \delta 7.31(\mathrm{~d}, J=6.9 \mathrm{~Hz}, 2 \mathrm{H}), 7.18(\mathrm{~m}$, 2H), 7.09 (t, $J=7.3 \mathrm{~Hz}, 1 \mathrm{H}), 6.13$ (d, $J=1.2 \mathrm{~Hz}, 1 \mathrm{H}), 4.33$ (d, $J=11.9 \mathrm{~Hz}, 1 \mathrm{H}), 4.27$ (q, $J=$ $11.9 \mathrm{~Hz}, 1 \mathrm{H}), 4.21(\mathrm{~m}, 1 \mathrm{H}), 4.13(\mathrm{dd}, J=10.9,1.5 \mathrm{~Hz}, 1 \mathrm{H}), 3.88$ (dd, $J=10.9,0.6 \mathrm{~Hz}, 1 \mathrm{H}), 1.98$ (dd, $J=15.3,10.8 \mathrm{~Hz}, 1 \mathrm{H}), 1.25$ (m, 1H), 1.21 (d, $J=6.3 \mathrm{~Hz}, 3 \mathrm{H}), 1.04$ (s, 3H), 0.96 (s, 3H), $0.48(\mathrm{~s}, 3 \mathrm{H}), 0.37(\mathrm{~s}, 3 \mathrm{H}) .{ }^{13} \mathrm{C} \mathrm{NMR}\left(100 \mathrm{MHz}, \mathrm{C}_{6} \mathrm{D}_{6}\right) \delta 154.1,139.1,135.1,128.5,127.9,127.6$, 72.0, 67.3, 50.5, 37.4, 34.0, 27.2, 25.8, 3.7, -0.4. IR (thin film) 2962, 1618 (w), 1454, 1371, $1248,1172,1084,830,784 \mathrm{~cm}^{-1}$. Anal. calc. for $\mathrm{C}_{18} \mathrm{H}_{28} \mathrm{O}_{2} \mathrm{Si}$ : C, 71.00; H, 9.27; Found: C, 70.87; $\mathrm{H}, 9.12$.

6-Benzyloxymethyl-3-(tert-butyldimethylsilyloxymethyl)-2,2-dimethyl-5, 6-dihydro-2H[1,2]oxasiline

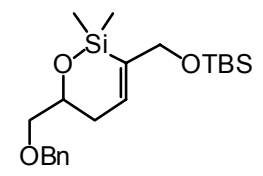


$\mathrm{R}_{\mathrm{f}} 0.61$ (80:20:1 pet. ether: EtOAc: MeOH). ${ }^{1} \mathrm{H}$ NMR (300 MHz, $\left.\mathrm{C}_{6} \mathrm{D}_{6}\right) \delta$ 7.08-7.28 (m, $\left.5 \mathrm{H}\right)$, $6.38(\mathrm{~m}, 1 \mathrm{H}), 4.36(\mathrm{~d}, J=1.7 \mathrm{~Hz}, 2 \mathrm{H}), 4.22(\mathrm{~s}, 2 \mathrm{H}), 4.17(\mathrm{~m}, 1 \mathrm{H}), 3.57$ (dd, $J=9.5,5.0 \mathrm{~Hz}, 1 \mathrm{H})$, $3.38(\mathrm{dd}, J=9.5,6.4 \mathrm{~Hz}, 1 \mathrm{H}), 2.19$ (m, 2H), 0.96 (s, 9H), 0.34 (s, 3H), 0.33 (s, 3H), 0.04 (s, 6H). ${ }^{13} \mathrm{C}$ NMR $\left(75 \mathrm{MHz}, \mathrm{C}_{6} \mathrm{D}_{6}\right) \delta 140.5,139.2,137.7,128.5,127.8,127.6,75.1,73.4,70.8,67.5$, 33.0, 26.1, 18.5, -0.1, -0.2, -5.3. IR (thin film) 2957, 2857, 1615 (w), 1472, 1362, 1252, 1105, $835 \mathrm{~cm}^{-1}$. Anal. calc. for $\mathrm{C}_{21} \mathrm{H}_{36} \mathrm{O}_{3} \mathrm{Si}_{2}: \mathrm{C}, 64.23 ; \mathrm{H}, 9.24$; Found: C, 64.13; H, 9.05 .

\section{2,2-Dimethyl-3-phenyl-1-oxa-2-sila-spiro[5.5]undec-3-ene}

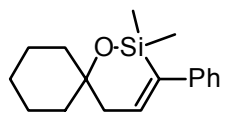

$\mathrm{R}_{\mathrm{f}} 0.54$ (20:1 pet. ether: ether). ${ }^{1} \mathrm{H}$ NMR (400 MHz, $\left.\mathrm{C}_{6} \mathrm{D}_{6}\right) \delta$ 7.03-7.22 (m, 5H), $6.53(\mathrm{t}, J=5.0$ $\mathrm{Hz}, 1 \mathrm{H}), 2.13$ (d, $J=5.0 \mathrm{~Hz}, 2 \mathrm{H}), 1.71-1.85(\mathrm{~m}, 4 \mathrm{H}), 1.52$ (m, 1H), 1.35 (m, 2H), 1.10-1.26 (m, 3H), 0.29 (s, 6H). ${ }^{13} \mathrm{C}$ NMR (100 MHz, $\left.\mathrm{C}_{6} \mathrm{D}_{6}\right) \delta$ 143.3, 141.5, 140.2, 128.8, 126.7, 126.5, 73.4, 41.5, 38.5, 26.4, 22.3, 1.3. IR (thin film) 3026 (w), 2933, 2859, 1596, 1491, 1444, 1251, 1010 $\mathrm{cm}^{-1}$. Anal. calc. for $\mathrm{C}_{17} \mathrm{H}_{24} \mathrm{OSi}$ C, 74.94; H, 8.88; Found: C, 74.76; H, 8.93.

2-Dimethylsilanyloxy-6,6,7-trimethyl-1,2,3,4,4a,6,9,9a-octahydro-5-oxa-6-silabenzocycloheptene

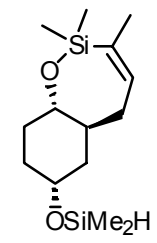

${ }^{1} \mathrm{H}$ NMR (400 MHz, $\left.\mathrm{C}_{6} \mathrm{D}_{6}\right) \delta 6.28(\mathrm{~m}, 1 \mathrm{H}), 4.84(\mathrm{sep}, J=2.7 \mathrm{~Hz}, 1 \mathrm{H}), 3.75(\mathrm{~m}, 1 \mathrm{H}), 3.46(\mathrm{ddd}, J$ $=11.1,11.1,4.2 \mathrm{~Hz}, 1 \mathrm{H}), 2.34(\mathrm{~m}, 1 \mathrm{H}), 2.19(\mathrm{~m}, 1 \mathrm{H}), 2.05(\mathrm{~m}, 1 \mathrm{H}), 1.99(\mathrm{dd}, J=16.8,7.6 \mathrm{~Hz}$, 1H), 1.89 (m, 1H), 1.73 (s, 3H), 1.68 (m, 1H), 1.59 (dddd, $J=13.1,3.4,3.4,3.4 \mathrm{~Hz}, 1 \mathrm{H}), 1.12$ (m, 1H), 0.96 (ddd, $J=13.1,13.1,2.2 \mathrm{~Hz}, 1 \mathrm{H}), 0.87$ (m, 1H), 0.36 (s, 3H), 0.27 (s, 3H), 0.11 (d, $J=2.7 \mathrm{~Hz}, 3 \mathrm{H}), 0.10(\mathrm{~d}, J=2.7 \mathrm{~Hz}, 3 \mathrm{H}) .{ }^{13} \mathrm{C}$ NMR $\left(100 \mathrm{MHz}, \mathrm{C}_{6} \mathrm{D}_{6}\right) \delta 141.7,138.1,79.6,68.0$, 40.7, 39.8, 37.4, 31.9, 30.3, 22.4, 0.9, -1.07, -1.13. IR (thin film) 2943, 2109, 1622 (w), 1444, 
1252, 1124, 1037, $897 \mathrm{~cm}^{-1}$. HRMS-EI $(\mathrm{m} / \mathrm{z}):[\mathrm{M}]^{+}$calcd for $\mathrm{C}_{14} \mathrm{H}_{24} \mathrm{O}_{2} \mathrm{Si}_{2}: 284.1628$; found: 284.1624 .

\section{Vinylsilane oxidation:}

Synthesis of $( \pm)-(1 R, 2 S)$-1-acetyloxy-2-(2-oxo-5-phenylpentyl)cyclohexane

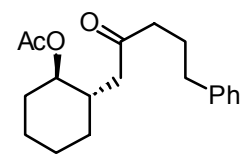

The vinyl silacycle (125 mg, $0.416 \mathrm{mmol})$ was taken up in DMF (4 mL) under air at rt. Solid $\mathrm{KHF}_{2}(98 \mathrm{mg}, 1.25 \mathrm{mmol})$ was added, followed by acetic anhydride $(1.2 \mathrm{~mL})$ and $30 \%$ aq. $\mathrm{H}_{2} \mathrm{O}_{2}$ $(1 \mathrm{~mL})$. After stirring for $14 \mathrm{hr}$ at rt, sat. aq. $\mathrm{NaHCO}_{3}(40 \mathrm{~mL})$ was added and the mixture stirred for 10 min until bubbling ceased. The mixture was extracted with EtOAc $(3 \times 15 \mathrm{~mL})$ and the organic extracts washed with brine $(15 \mathrm{~mL})$, dried over $\mathrm{Na}_{2} \mathrm{SO}_{4}$, and concentrated in vacuo. The crude alcohol was then taken up in $\mathrm{CH}_{2} \mathrm{Cl}_{2}(2 \mathrm{~mL})$ and treated with pyridine $(134 \mu \mathrm{L}, 1.66$ mmol). After cooling to $0{ }^{\circ} \mathrm{C}$, acetyl chloride $(59 \mu \mathrm{L}, 0.83 \mathrm{mmol})$ was then added and the reaction mixture allowed to stir for 15 minutes. Sat. aq. $\mathrm{Na}_{2} \mathrm{SO}_{4}(15 \mathrm{~mL})$ is then added, and the mixture extracted with EtOAc $(3 \times 15 \mathrm{~mL})$. The extracts were washed with $0.5 \mathrm{M} \mathrm{HCl}(10 \mathrm{~mL})$, sat. aq. $\mathrm{Na}_{2} \mathrm{SO}_{4}(10 \mathrm{~mL})$, and brine $(10 \mathrm{~mL})$, then dried over $\mathrm{Na}_{2} \mathrm{SO}_{4}$, and concentrated in vacuo. The crude product was then purified on silica gel $(20: 1$, then 10:1 pet. ether: EtOAc) which provided $78 \mathrm{mg}(62 \%)$ of the desired acetoxy ketone as a colorless oil.

$\mathrm{R}_{\mathrm{f}} 0.44$ (80:20:1 pet. ether: EtOAc: $\left.\mathrm{MeOH}\right) .{ }^{1} \mathrm{H}$ NMR (500 MHz, $\left.\mathrm{CDCl}_{3}\right) \delta$ 7.27-7.30 (m, $\left.2 \mathrm{H}\right)$, 7.16-7.21 (m, 3H), 4.43 (ddd, $J=10.3,10.3,4.4 \mathrm{~Hz}, 1 \mathrm{H}), 2.61$ (t, $J=6.6 \mathrm{~Hz}, 2 \mathrm{H}), 2.48$ (dd, $J=$ 16.2, $4.4 \mathrm{~Hz}, 1 \mathrm{H}), 2.39$ (t, $J=7.4 \mathrm{~Hz}, 2 \mathrm{H}), 2.16(\mathrm{dd}, J=16.2,8.3 \mathrm{~Hz}, 1 \mathrm{H}), 2.06(\mathrm{~m}, 1 \mathrm{H}), 1.99(\mathrm{~s}$, 3H), $1.98(\mathrm{~m}, 1 \mathrm{H}), 1.89(\mathrm{~m}, 2 \mathrm{H}), 1.80(\mathrm{~m}, 1 \mathrm{H}), 1.74(\mathrm{~m}, 1 \mathrm{H}), 1.57-1.64(\mathrm{~m}, 2 \mathrm{H}), 1.20-1.35(\mathrm{~m}$, $3 \mathrm{H}), 0.99$ (dddd, $J=12.2,12.2,12.2,2.9 \mathrm{~Hz}, 1 \mathrm{H}) .{ }^{13} \mathrm{C} \mathrm{NMR}\left(125 \mathrm{MHz}, \mathrm{CDCl}_{3}\right) \delta 209.9,170.8$, 141.6, 128.44, 128.38, 126.0, 76.5, 46.2, 42.5, 38.2, 35.1, 31.8, 31.3, 25.2, 25.0, 24.5, 21.2. IR (thin film) 2935, 2859, 1732 (s), 1732 (s), 1713 (s), 1452, 1372, 1242 (s), $1030 \mathrm{~cm}^{-1}$. Anal. calc. for $\mathrm{C}_{19} \mathrm{H}_{26} \mathrm{O}_{3}$ : C, 75.46; H, 8.67; Found: C, 75.29; H, 8.53. 
Palladium-catalyzed Cross-coupling

Synthesis of (Z)-6-Phenyl-5-hepten-1-ol

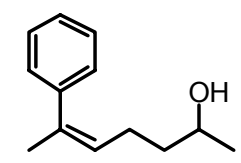

5-Heptyne-2-ol (100 $\mathrm{mg}, 0.892 \mathrm{mmol})$ was subjected to the standard intramolecular hydrosilylation conditions above to afford $145 \mathrm{mg}(96 \%)$ of the desired silacyle (145 $\mathrm{mg}, 96 \%)$ contaminated with small amounts of polymeric material after filtration through a small plug of Florisil. This material was used directly in the next reaction $\left({ }^{1} \mathrm{H}\right.$ NMR $\left(400 \mathrm{MHz}, \mathrm{C}_{6} \mathrm{D}_{6}\right) \delta 6.23$ $(\mathrm{m}, 1 \mathrm{H}), 3.96(\mathrm{~m}, 1 \mathrm{H}), 2.28(\mathrm{~m}, 1 \mathrm{H}), 2.02(\mathrm{~m}, 1 \mathrm{H}), 1.71(\mathrm{~d}, J=1.5 \mathrm{~Hz}, 3 \mathrm{H}), 1.54(\mathrm{~m}, 2 \mathrm{H}), 1.15$ $(\mathrm{d}, J=6.1 \mathrm{~Hz}, 3 \mathrm{H}), 0.27(\mathrm{~s}, 3 \mathrm{H}), 0.20(\mathrm{~s}, 3 \mathrm{H}))$.

The silacycle (35 mg, $0.20 \mathrm{mmol})$ was taken up in THF $(0.40 \mathrm{~mL})$ at $\mathrm{rt}$ under an Ar atmosphere. The solution was treated sequentially with iodobenzene $(35 \mu \mathrm{L}, 0.30 \mathrm{mmol})$ and then TBAF (0.40 mL, $0.40 \mathrm{mmol}, 1.0 \mathrm{M}$ in THF). Solid $\mathrm{Pd}_{2} \mathrm{dba}_{3} \cdot \mathrm{CHCl}_{3}(9.5 \mathrm{mg}, 0.005 \mathrm{mmol})$ was then introduced and the solution was brought to $55^{\circ} \mathrm{C}$. After stirring for $6 \mathrm{hr}$, the solution was allowed to cool, and was then filtered though a short plug of silica, eluting with pure ether, and concentrated in vacuo. The resulting residue was purified by silica gel chromatography (10:1, then $4: 1$ pet. ether: ether) to provide the desired trisubstituted olefin as a pale yellow oil (32.5 $\mathrm{mg}, 83 \%)$.

${ }^{1} \mathrm{H}$ NMR $\left(400 \mathrm{MHz}, \mathrm{CDCl}_{3}\right) \delta 7.33(\mathrm{dd}, J=7.0,7.0 \mathrm{~Hz}, 2 \mathrm{H}), 7.24(\mathrm{~m}, 1 \mathrm{H}), 7.17(\mathrm{~d}, J=7.0 \mathrm{~Hz}$, 2H), $5.46(\mathrm{td}, J=7.3,1.2 \mathrm{~Hz}, 1 \mathrm{H}), 3.73(\mathrm{~m}, 1 \mathrm{H}), 2.06(\mathrm{~m}, 2 \mathrm{H}), 2.02(\mathrm{~s}, 3 \mathrm{H}), 1.48(\mathrm{~m}, 2 \mathrm{H}), 1.30$ (br OH, $1 \mathrm{H}), 1.10(\mathrm{~d}, J=6.0 \mathrm{~Hz}, 3 \mathrm{H}) .{ }^{13} \mathrm{C} \mathrm{NMR}\left(100 \mathrm{MHz}, \mathrm{CDCl}_{3}\right) \delta 141.9,136.8,128.1,127.9$, 126.9, 126.5, 67.6, 39.4, 25.6, 25.4, 23.2. IR (thin film) 3358 (br, OH), 3022 (w), 2967 (s), 2926 (s), $1600(\mathrm{w}), 1435,1374,1130,1080,763,701$.

Crossover Experiment 


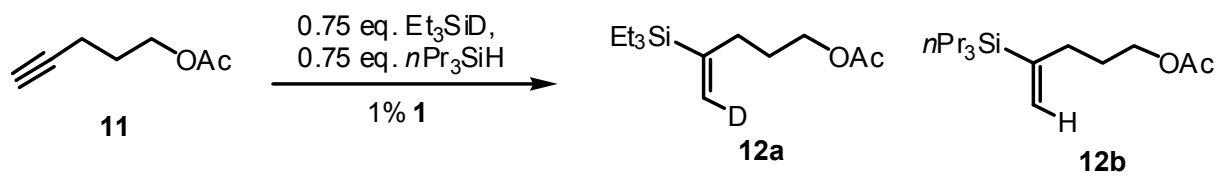

5-Acetyloxy-1-pentyne (38 $\mu \mathrm{L}, 0.29 \mathrm{mmol})$ was taken up in $\mathrm{CH}_{2} \mathrm{Cl}_{2}(0.60 \mathrm{~mL})$ at $0{ }^{\circ} \mathrm{C}$ under $\mathrm{Ar}$. The solution was treated with equimolar amounts of $\operatorname{HSi}(n \operatorname{Pr})_{3}(47 \mu \mathrm{L}, 0.23 \mathrm{mmol})$ and $\mathrm{DSiEt}_{3}$ (36 $\mu \mathrm{L}, 0.23 \mathrm{mmol})$. The solution was next treated with solid $\left[\mathrm{Cp}{ }^{*} \mathrm{Ru}(\mathrm{MeCN})_{3}\right] \mathrm{PF}_{6}(1.3 \mathrm{mg}$, $0.0026 \mathrm{mmol}$ ) and the reaction vessel allowed to warm to rt. After $20 \mathrm{~min}$, the alkyne was consumed as determined by TLC analysis, and the crude reaction mixture was filtered through a plug of Florisil, washing with ether. The crude solution thus obtained was analyzed directly by GC/MS. Peaks arising from $(n \mathrm{Pr})_{3} \mathrm{SiH}$ and $\mathrm{Et}_{3} \mathrm{SiD}$ were of similar intensity, indicating that, as expected, the two very similar silanes reaction at comparable rates. GC/MS data is given in the figures at the end of the supporting information. Figure S1 shows a GC/MS trace from a typical hydrosilylation without deuterium incorporation with an MS for the major product peak. Figure S2 shows a similar GC/MS, this time demonstrating complete D incorporation upon use of $\mathrm{Et}_{3} \mathrm{SiD}$ (note $[\mathrm{M}+1]^{+}$, no $\mathrm{M}^{+}$peaks). Figure $\mathrm{S} 3$ shows results from the crossover experiment, with the two silane products clearly separating in the GC. S3 gives an MS for the first peak from hydrosilylation with $\mathrm{Et}_{3} \mathrm{SiD}$ without any $\mathrm{M}^{+}$. Figure $\mathrm{S} 4$ shows the same $\mathrm{GC}$ trace, with MS data from the second peak resulting from $(n \mathrm{Pr})_{3} \mathrm{SiH}$.

Intermolecular Hydrosilylation of Terminal Alkyne Stereochemistry

2-triethylsilyl-5-acetyloxy-1-pentene- $d^{2}$

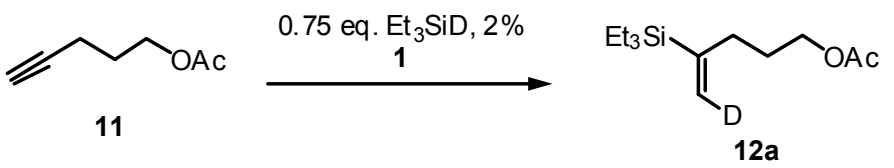

5-Acetyloxy-1-pentyne (34 $\mu \mathrm{L}, 0.26 \mathrm{mmol})$ was taken up in $\mathrm{CH}_{2} \mathrm{Cl}_{2}(0.60 \mathrm{~mL})$ at $0{ }^{\circ} \mathrm{C}$ under $\mathrm{Ar}$. The solution was treated with $\mathrm{DSiEt}_{3}(50 \mu \mathrm{L}, 0.31 \mathrm{mmol}$, Aldrich, 97\% D). The solution was next treated with solid $\left[\mathrm{Cp}^{*} \mathrm{Ru}(\mathrm{MeCN})_{3}\right] \mathrm{PF}_{6}(2.6 \mathrm{mg}, 0.0052 \mathrm{mmol})$ and the reaction vessel allowed to warm to rt. After $1 \mathrm{hr}$, the alkyne was consumed as determined by TLC analysis, and the crude reaction mixture was concentrated and applied directly to a silica gel column (eluent 20:1 pet. ether: ether) to afford the desired selectively deuterated vinylsilane. NMR analysis 
indicated full deuterium incorporation at the trans position indicated in the reaction above. Isomeric purity is conservatively estimated at $\geq 97: 3$. Relevent ${ }^{1} \mathrm{H} \mathrm{NMR}$ : $\left(300 \mathrm{MHz}, \mathrm{CDCl}_{3}\right) \delta$ $5.65(\mathrm{~s}, 1 \mathrm{H},(E)$ proton trans to silicon, $1 \mathrm{H}) 5.33(\mathrm{~s}, 1 \mathrm{H},(Z)$ proton cis to silicon, $1 \mathrm{H})$. The assignment of olefinic protons was made through nOe data:

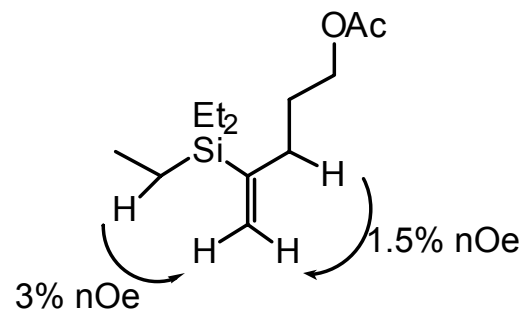

References:

(1) Steinmetz, B.; Schenk, W. A. Organometallics 1999, 18, 943-946.

(2) Trost, B. M.; Ball, Z. T. J. Am. Chem. Soc. 2001, 123, 12726-12727. 
FigureS1. GC/MS of typical crude hydrosilylation reaction.

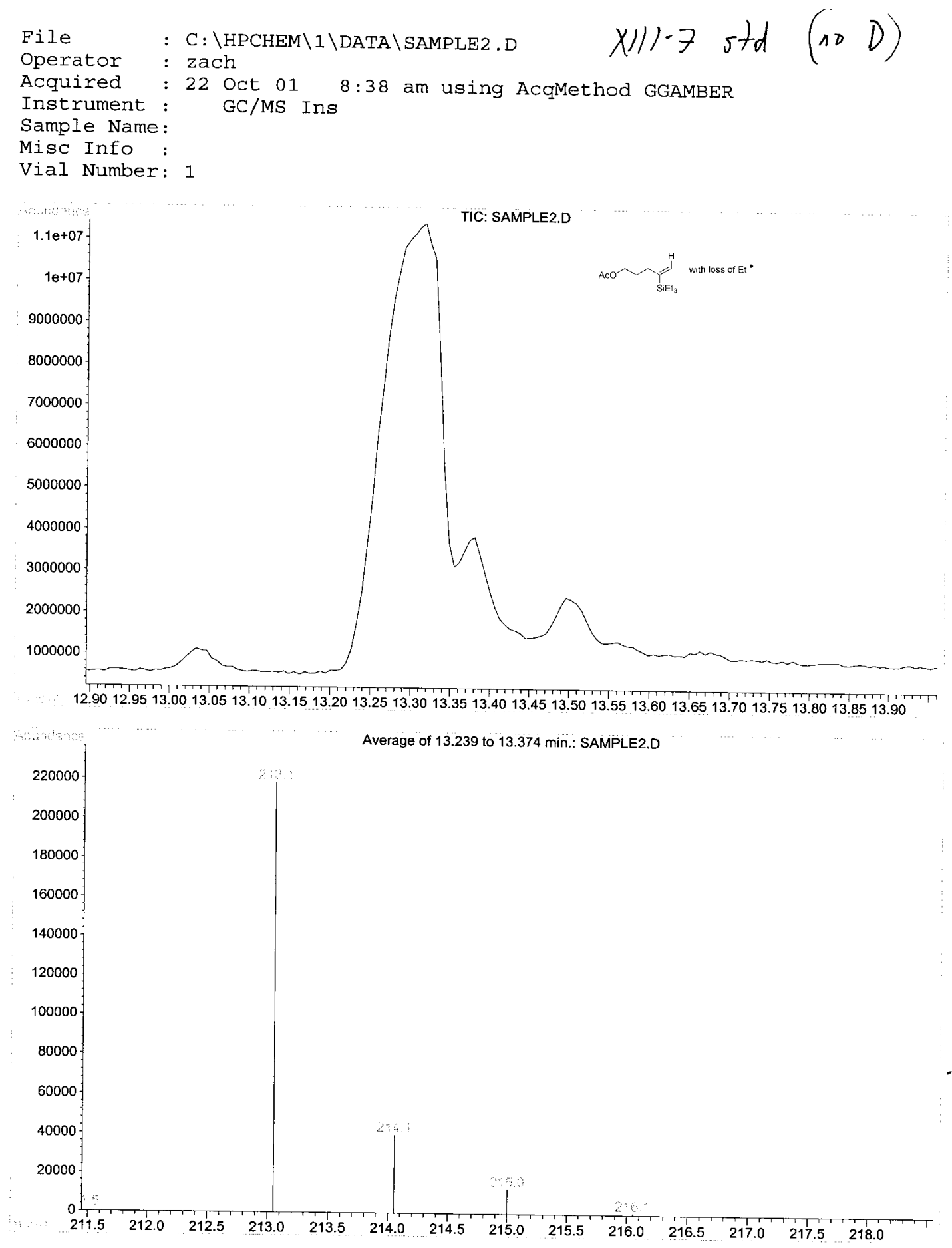


Figure S2. GC/MS of reaction with deuterated silane. No [M-1] peak indicates no scambling with alkyne $\mathrm{C}-\mathrm{H}$ of starting material.

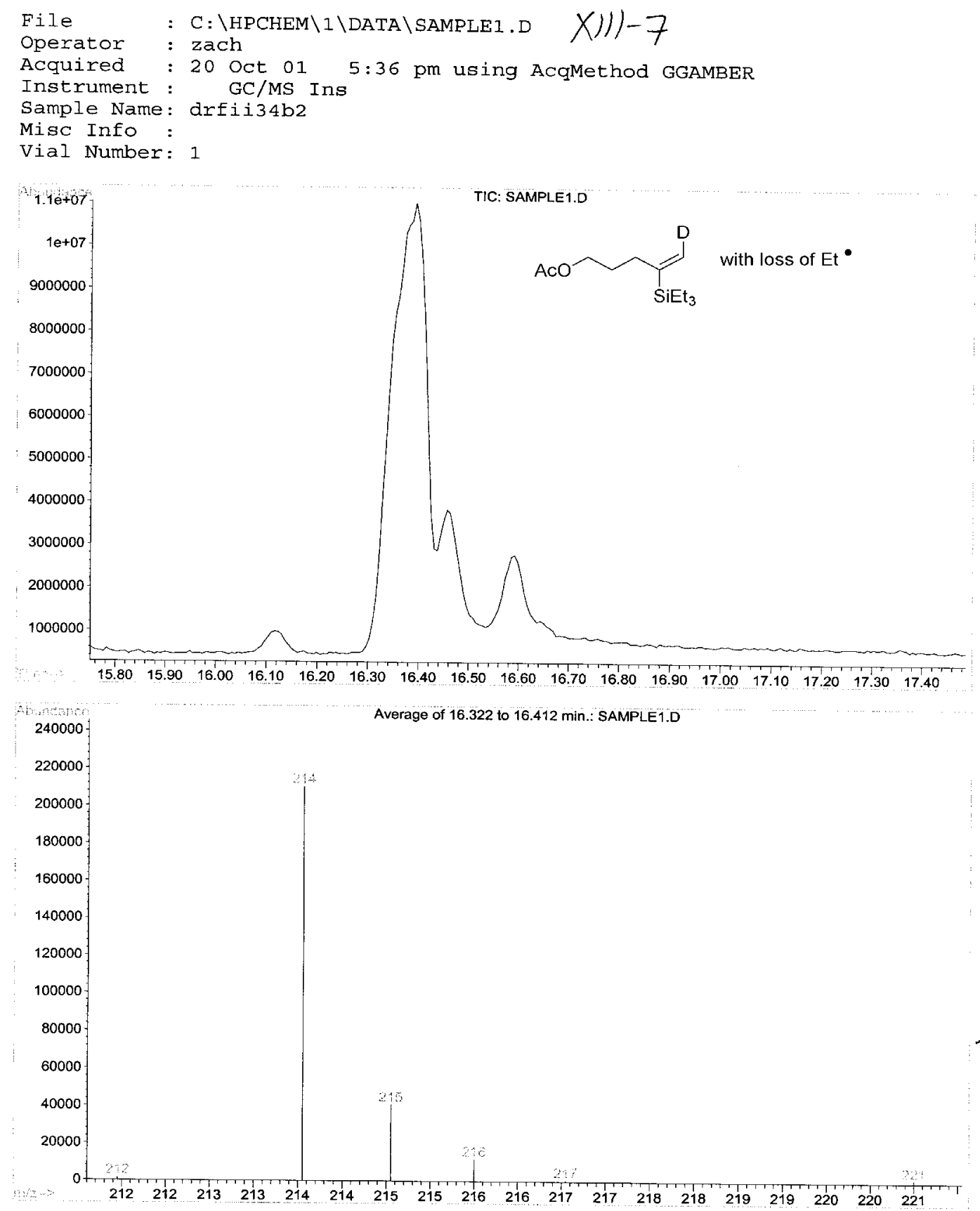


Figure S3. GC/MS trace of crossover experiment with expansion on MS of peak corresponding to $\mathrm{DSiEt}_{3}$ product. Lack of [M-1] peak indicates no crossover with competing $\mathrm{HSi}(n \operatorname{Pr})_{3}$.

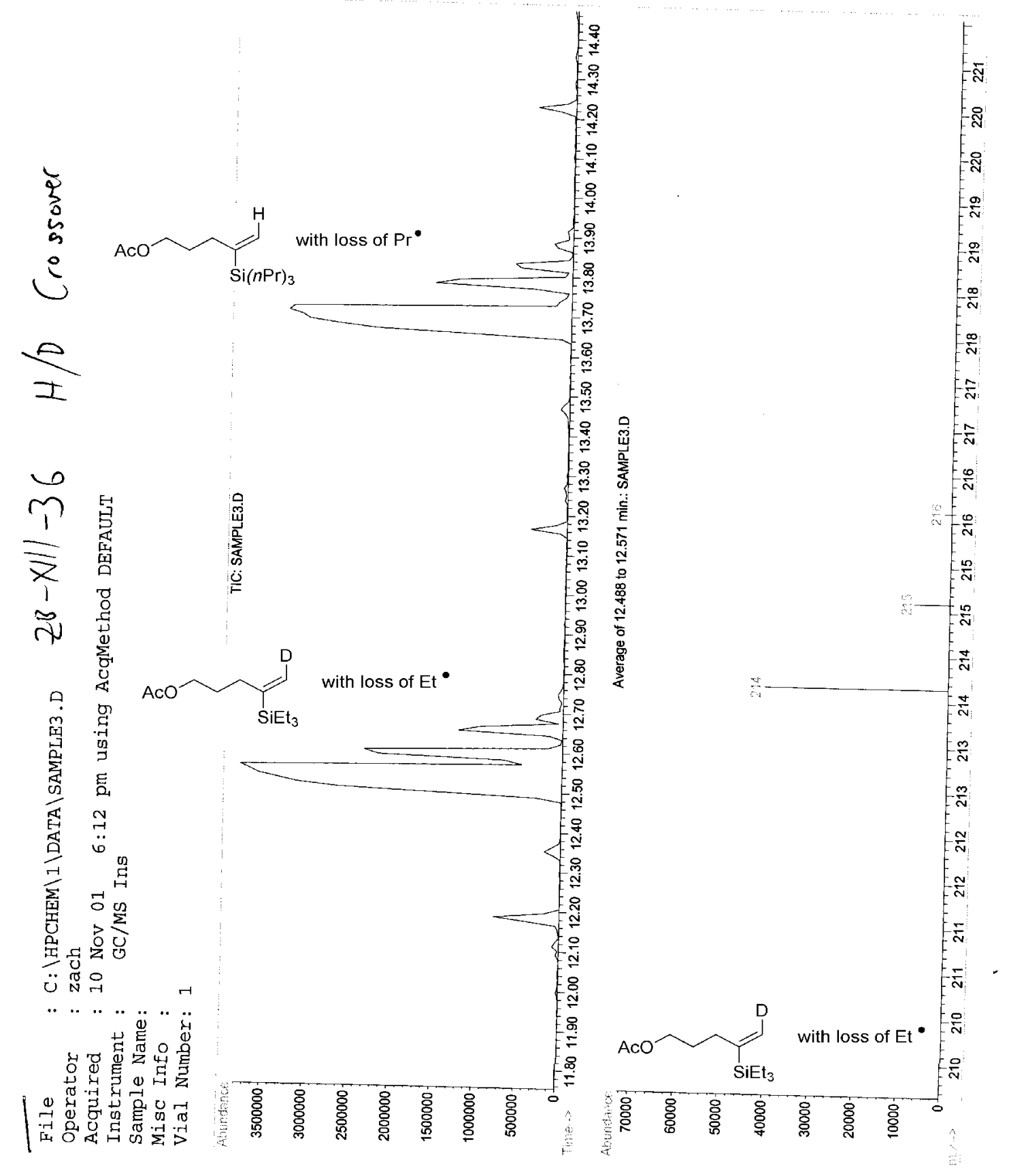


Figure S4. Repeated GC/MS trace from fig. S3, with MS expansion on $\mathrm{HSi}(n \mathrm{Pr})_{3}$.

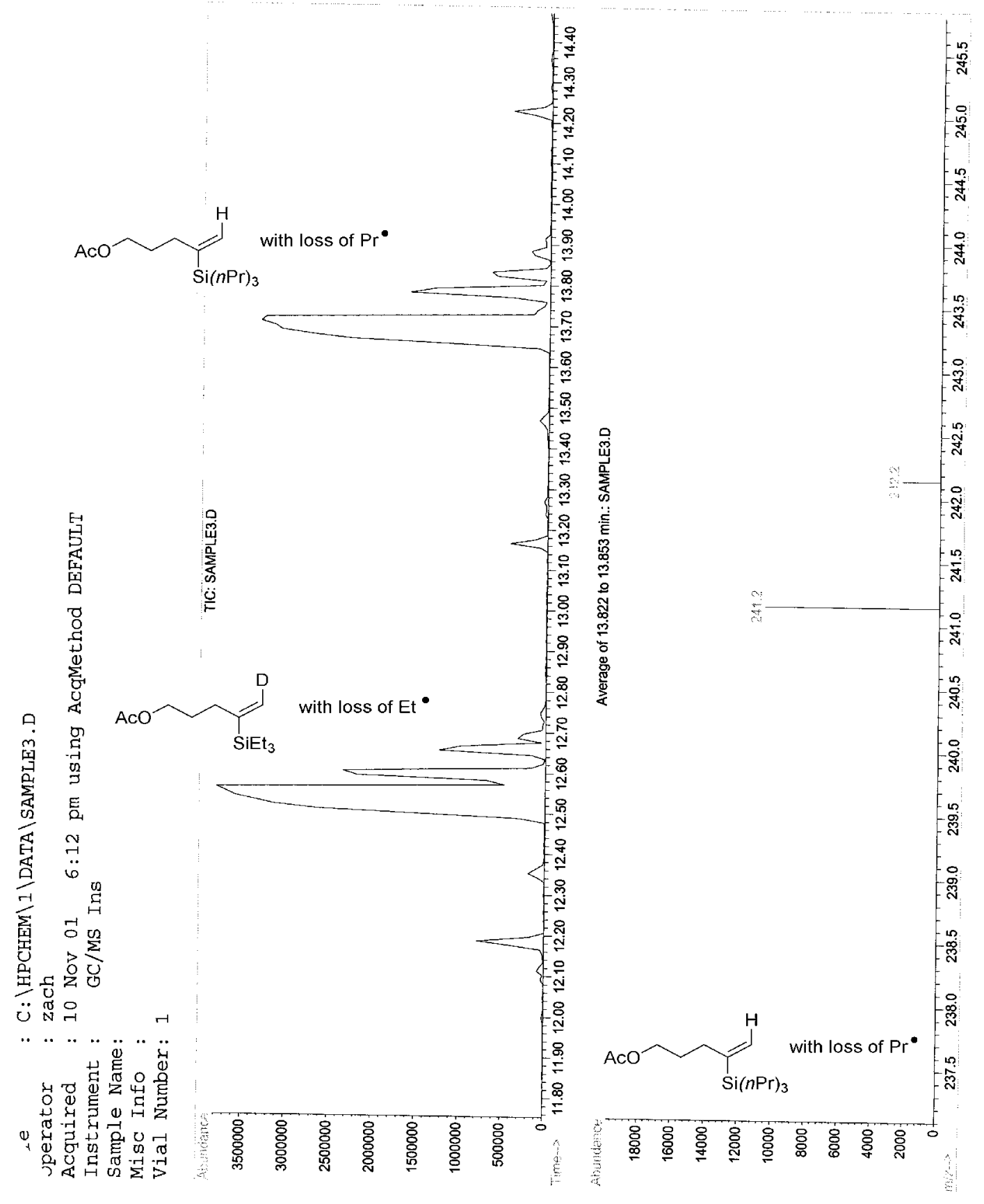

